\title{
Viscosity Measurement of Olive Oil under Pressure
}

\section{Pawlicki LT*, Urbański MK, Tefelski D and Siegoczyński RM}

Faculty of Physics, Warsaw University of Technology, Koszykowa 75, 00-662 Warsaw, Poland

*Corresponding author: Pawlicki LT, Faculty of Physics, Warsaw University of Technology, Koszykowa 75, 00-662 Warsaw, Poland, Email: leszek.pawlicki@pw.edu.pl

\section{Research Article \\ Volume 6 Issue 5}

Received Date: September 06, 2021

Published Date: September 22, 2021

DOI: $10.23880 /$ fsnt-16000275

\section{Abstract}

This article presents changes in the viscosity of olive oil during compression. The test was carried out indirectly by measuring the dependence of the resonance frequency of the piezoelectric immersed in olive oil on pressure. For this purpose, for successive pressures, the resonance curves were read and the values of the characteristic frequencies were determined. Viscosity changes were analysed and related to the compression and crystallization taking place in the tested substance. During this research, a phase transition from the liquid phase to the alpha crystalline phase was detected, during which the resonant frequency of the tested piezoelectric reached a minimum and the viscosity related to this frequency reached a maximum. The measurement method developed in this paper can be used to detect the phase transitions of oils subjected to pressure. This may find application in the oil production and high-pressure food preservation industries for which this knowledge is essential for the safe and trouble-free use of their machines.

Keywords: Viscosity; Olive Oil; High Pressure

\section{Introduction}

In the resonance method, as well as in the ultrasonic method, a piezoelectric is used immersed in the test substance contained in a pressure chamber. The resonance method is based on the search for the resonant frequency of piezoelectric vibrations in function of parameters (temperature, pressure) [1,2]. The value of this frequency depends on the viscosity and elasticity of the medium in which the piezoelectric vibrates. These parameters, on the other hand, change during compression and during the phase transitions taking place in the substances [3]. Therefore, the knowledge of the dependence of the resonance frequency on the pressure allows for the analysis of changes occurring in the tested substance during these transformations. Olive oil is an mixture of triglycerides. This cause that it should have characteristic for them phase transitions and crystal form like e. g. alpha form [3] (Figure 1).

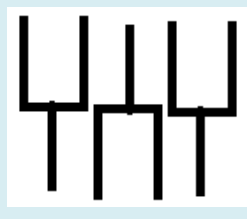

Figure 1: Alpha crystal form.

Viscosities have been measured for some triglycerides in earlier articles $[4,5]$. Phase transitions in oils have been earlier detected by many different methods and described in earlier works for: castor oil [6-10], rapeseed oil [11], soy oil [12], sunflower oil $[13,14]$. That articles show that there should be phase transitions in olive oil to. So investigation on phase transitions in olive oil have been made by using ultrasonic method $[14,15]$ and electric method [16]. Now is the time to investigate its mechanical properties using the resonance method described in this paper. 


\section{Examined Substance}

The examined substance in our research was Virgin Olive
Oil produced by Star (USA) with triglyceride composition shown in Table 1.

\begin{tabular}{|c|c|c|c|c|c|c|c|c|c|c|c|}
\hline Content [\%] & 76.8 & 11.2 & 5.9 & 3.4 & 1 & 0.7 & 0.4 & 0.3 & 0.1 & 0.1 & 0.1 \\
\hline Fatty Acid & C18:1 & C16:0 & C18:2 & C18:0 & C16:1 & C18:3 & C20:0 & C20:1 & C17:0 & C17:1 & C22:0 \\
\hline
\end{tabular}

Table 1: Molecular composition of olive oil.

This olive oil has been chosen for our experiment because it was an example of a typical good quality olive oil. Its composition was determined by chromatographic methods.

\section{Measurement System}

The basic element of our system was a pressure chamber, inside which the actual measuring system was flooded with the tested olive oil (Figure 2).

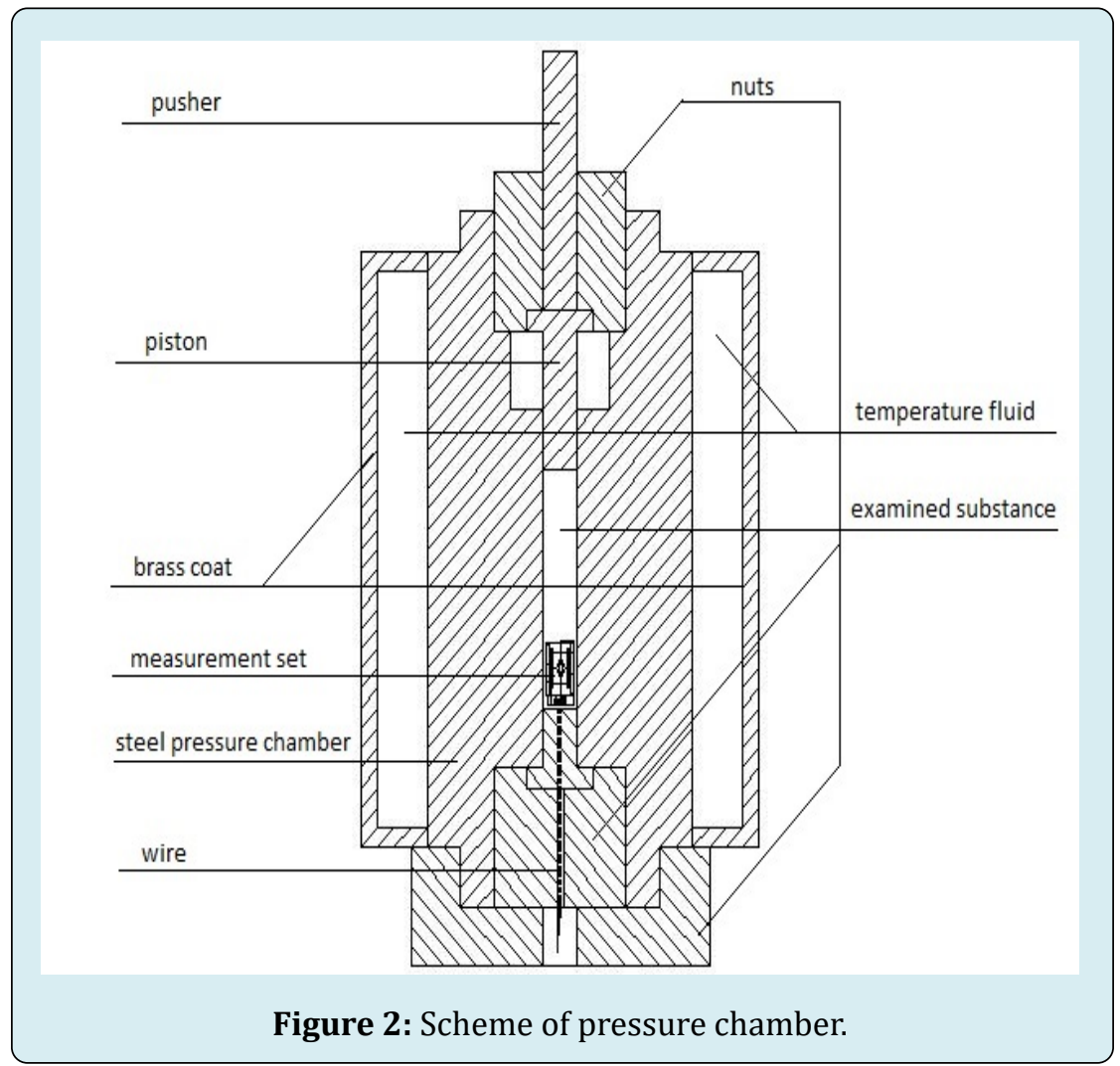

The main part of the pressure chamber was a steel pipe $23 \mathrm{~cm}$ long. The tube had an outer diameter of $56 \mathrm{~mm}$ and an inner diameter of $17 \mathrm{~mm}$. It was made of $40 \mathrm{H}$ steel. Additionally, the pipe was covered on the side with a brass casing. An air pocket was created between the steel pipe and the brass jacket. From the bottom, the steel pipe was closed by a steel culvert plug. After inserting the inner part of the measuring system into the chamber, the opening was closed with the above-mentioned culvert plug. The plug was pressed inside the chamber, and the connection was sealed with a brass pressure gasket and a rubber gasket. Above this plug, a steel pressure plug was screwed in from the inside of the pipe with a thread and tightened with a wrench, which allowed for sealing the bottom side of the chamber. Additionally, in order to ensure greater safety, a safety plug was screwed onto the outer part of the chamber tube by means of a thread. From above, the test substance was poured into the steel pipe (constituting the main part of the pressure chamber), leaving part of its length empty for insertion of the piston fragment. Then the pipe was closed by pressing a movable steel piston made of NC 10 steel into it, and the connection was sealed with a brass pressure gasket and a rubber gasket. Above the piston, from the inside of the pipe, a special steel locking plug is screwed in with a thread. This plug, thanks to its construction, did not allow the piston to slide out of the chamber and unseal the system. There was an opening 
in the cork, allowing the steel pusher to pass through it. The pusher mediated the action of the hydraulic press on the piston surface. In this way, a sealed pressure chamber was created inside the steel pipe, filled with the substance to be tested, which could penetrate into the part of the measuring system located inside it. This inside part of the measuring system consisted of an anechoic chamber closed inside a brass cylindrical capacitor, which contained a piezoelectric zirconium crystal resonator PZT with mechanical resonant near $150 \mathrm{kHz}$. The outer cover of the capacitor was $14.8 \mathrm{~mm}$ in outer diameter, $1 \mathrm{~mm}$ thick, and $40 \mathrm{~mm}$ long. The inner cover of the capacitor was $11.8 \mathrm{~mm}$ in outer diameter, $1 \mathrm{~mm}$ thick, and $35 \mathrm{~mm}$ long. The piezoelectric used had a diameter of $5 \mathrm{~mm}$ and a length of $10 \mathrm{~mm}$.

The inside measuring system also included a manganinium resistor in the form of a coreless coil for indirect pressure measurement (dependence of manganin resistance on pressure has a linear character for the pressure range we are testing). It also included a " $\mathrm{T}$ " type thermocouple for temperature measurement (Figure 3).

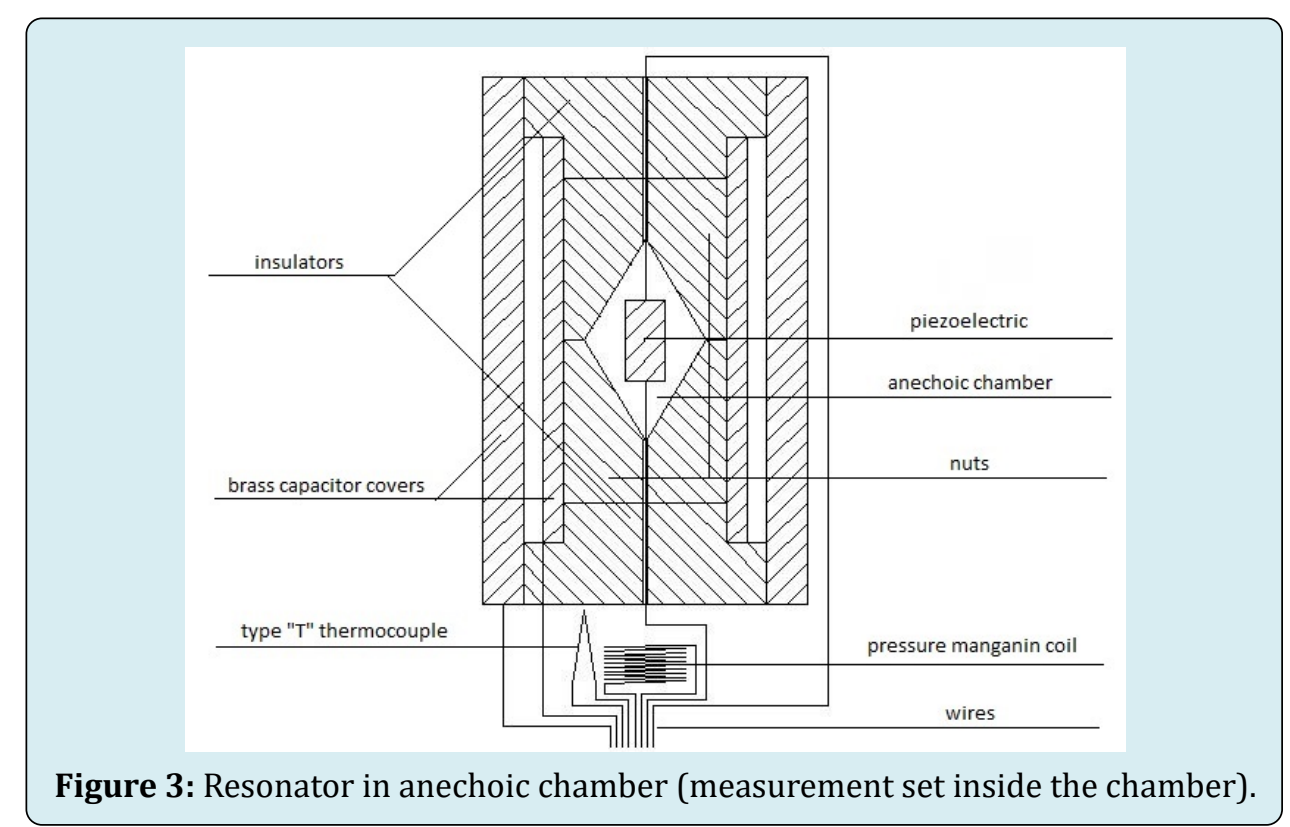

The measuring system was connected to a set of gauges placed outside the pressure chamber by wires led through a special pressure plug fixed in the bottom of the chamber. The data from inner measuring system have been read by meters and then transferred to a computer where they were collected by a special program made in the Lab View environment. This program also made direct time measurements. The pressure was introduced into the chamber by a stepwise method (by force exerted on the piston closing the chamber from above) by a manual hydraulic press. The stepped method allowed for the removal of excess heat from the pressure chamber resulting from compression and allowed tested substance to obtain a thermodynamic equilibrium. For each step of pressure we have measured the resonance curve for viscous liquid. In order to reduce the effect of the reflection of acoustic waves from the pressure chamber, the piezoelectric crystal was placed in an anechoic chamber shaped like two cones folded together with bottom.

The measuring system to determine the resonance curve consist of signal generator HAMEG HM8131, RMS voltage meter HP 427A and DC digital multimeter HP 34970a.
The measurement method consists in measuring the electric current (by measuring the voltage on series resistor) in the piezoelectric as a function of frequency at a constant voltage of the generator (Figure 4). The equivalent circuit models of PZT piezoelectric are the same as normal of quartz [17].

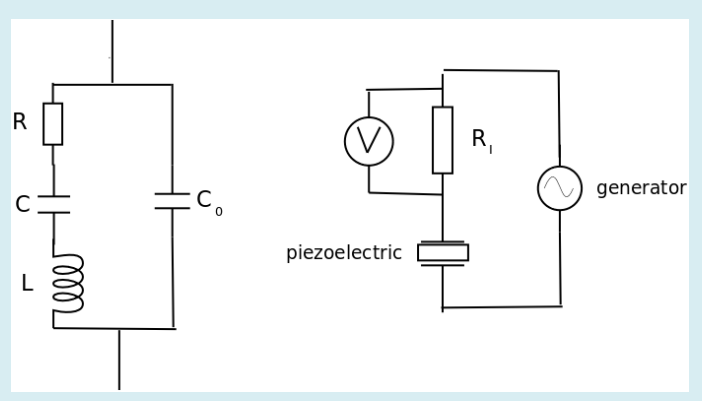

Figure 4: Equivalent circuit model of piezoelectric (left), circuit for measurement of resonances curve (right).

An example of the piezoelectric frequency response is shown in Fig. 5. The shape of this curve can be explained on 
the basis of the equivalent diagram of piezoelectric given on figure 4. Elements of the equivalent circuit represent the following responses: inductance L- mass of the piezoelectric material, resistance $\mathrm{R}$ - forces of resistance to motion (viscosity of the medium and mechanical losses), capacity C - material elasticity, capacity $\mathrm{C}_{0}$ - electric capacity of the piezoelectric resonance frequency is approximately equal to the intersection of the curve with the trend line (Figure 5)

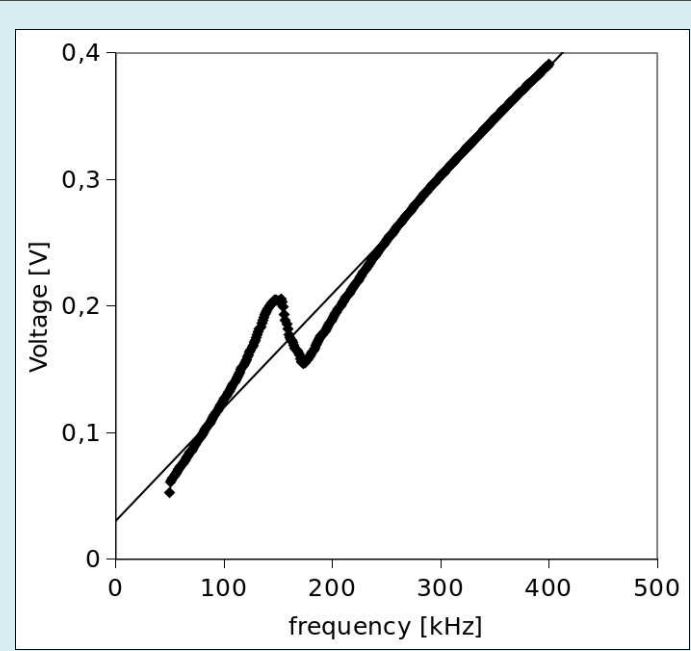

Figure 5: Piezoelectric frequency response (resonance curve). The resonance frequency is equal to the intersection of the curve with the trend line

\section{Results and Discussion}

The resonant frequency depends on the capacitance; inductance and resistance of the piezoelectric equivalence circuit (Figure 4). The resistance describes the losses in the resonance circuit and is proportional to the viscosity of the medium in which the vibrations take place (in analogy to situation described in [1] for quatz). The resonance frequency increases with the modulus of elasticity (in the equivalent diagram represented by inductance) and decreases with the medium damping (proportional to the viscosity) and represented by resistance.

Figure 5 shows the dependence of the piezoelectric resonance frequency in oil as a function of the pressure obtained in the chamber. The value of the resonance frequency was determined on the basis of the frequency characteristic (Figure 4) using the numerical method.In the range up to $200 \mathrm{MPa}$, the resonance frequency decreases, which we interpret as an increase in viscosity. At a pressure of $200 \mathrm{MPa}$, a phase transition begins and an increasing part of the oil crystallizes into alpha forms, therefore elastic forces appear instead of viscous forces and the resonance frequency increases (Figure 6).

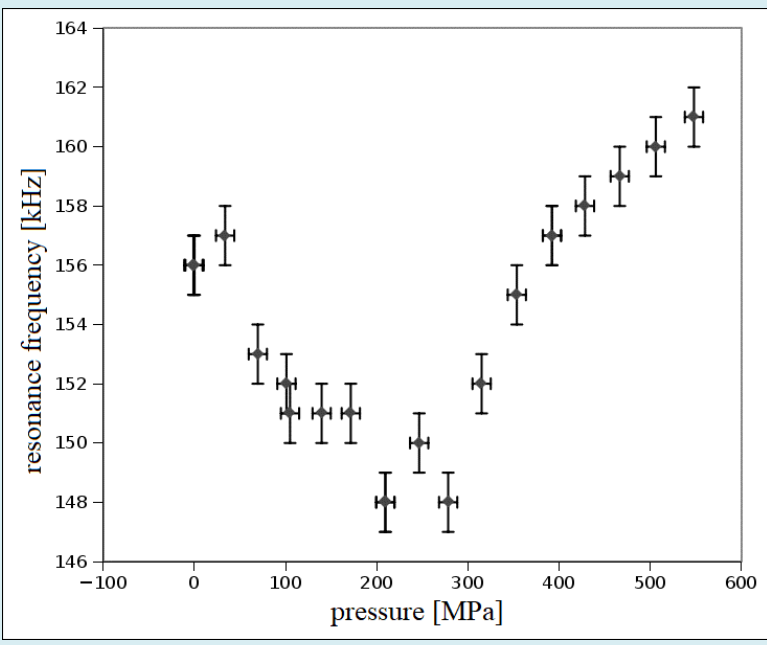

Figure 6: Graph of the dependence of the resonance frequency on the pressure.

The observed course of the dependence of the resonance frequency confirms the occurrence of the phase transition. These phase transitions have been observed in earlier work by electric methods (measuring electric resistivity, electric permittivity and impedance) [16].

\section{Summary and Conclusion}

The paper presents qualitative measurements of changes in the viscosity of olive oil and the elasticity coefficient of the solid phase in the pressure function in the range up to $600 \mathrm{MPa}$. A vibrating piezoelectric was used for the measurements, the resonance frequency of which depends on the viscosity of the medium in which it is immersed. Such a measurement system allows for the conclusion that the viscosity increases with pressure, which is observed as a decrease in the resonance frequency. After reaching the minimum, the resonant frequency increases, which we interpret as crystallization, which causes an increase in the resonance frequency of the vibrating piezoelectric. The crystallization process in the case of oils is a continuous change, which means that the crystallization takes place gradually and not in the entire volume at once.

The moment and the corresponding pressure for which the change in behaviour of the test substance occurs in this article coincide with the changes following for the electrical quantities following the same phase transition for olive oil presented in an earlier work [16]. The electric permittivity have point of inflection and the electric resistivity have maximum in this work when in our work the resonance frequency reach minimum value and viscosity reach maximum value[16]. It happens during the crystallization of the oil. 


\section{Food Science \& Nutrition Technology}

The knowledge contained in this article can therefore be applied in the industry of oil production and high-pressure food preservation, for which information about viscosity changes is crucial for the proper operation of machines.

\section{References}

1. Daridon JL, Cassiède M, Paillo JH, Pauly J (2011) Viscosity measurements of liquids under pressure by using the quartz crystal resonators. Review of Scientific Instr 82: 095114.

2. Steinem C, Janshoff A (2007) Piezoelectric Sensors.

3. Sato K (1999) Solidification and phase transformation behaviour of food fats - review. Fett/Lipid 101(12): 467 474.

4. Siegoczyński RM, Rostocki AJ, Kiełczyński P, Szalewski M (2008) A viscosity measurement during the high pressure phase transition in triolein. Journal of Physics: Conference Series 121(14): 142010.

5. Rostocki AJ, Siegoczyński RM, Kieczyński P, Szalewski M (2010) An application of love SH waves for the viscosity measurement of triglycerides at high pressures, High Pressure. High Pressure Research 30(1): 88-92.

6. Siegoczyński RM, Jędrzejewski J, Wiśniewski R (1989) Long time relaxation effect of liquid castor oil under high pressure condition. High Pressure Research 1(4): 225233.

7. Siegoczyński RM, Jędrzejewski J, Wiśniewski R (1991) The Influence of Pressure on the Refractive Index of Castor Oil up to 0.8 GPa. High Pressure Research 8(1-3): 439-441.

8. Wiśniewski R, Jędrzejewski J, Siegoczyński RM, Tkacz A (1994) Volume changes of castor oil during its transformation to the high pressure phase. High Pressure Research 11(6): 385-391.

9. Wiśniewski R, Jędrzejewski J, Siegoczyński RM (1994) Dielectric permittivity and dielectric loss of castor oil during its transformation to the high pressure phase. High Pressure Research 13: 41-45.

10. Wiśniewski R, Siegoczyński RM, Rostocki AJ (2005) Viscosity measurement of some castor oil based mixtures under high pressure conditions. High Pressure Research 25(1): 63-70.

11. Rostocki AJ, Wiśniewski R, Wilczyńska T (2007) High pressure phase transition in rape-seed oil. Mol Liq 135(8): 120-122.

12. Rostocki AJ, Kościesza R, Tefelski DB, Kos A, Siegoczyński RM, Chruściński L (2007) Pressure induced phase transition in soy oil. High Pressure Research 27(1): 4345.

13. Guinda A, Dobarganes MC, Ruiz-Mendez MV, Mancha M (2003) Chemical and physical properties of a sunflower oil with high levels of oleic and palmitic acids. Eur J Lipid Sci Technol 105(3-4): 130-137.

14. Rostocki AJ, Tarakowski R, Kiełczyński P, Szalewski M, Balcerzak A, Ptasznik S (2013) The ultrasonic investigation of phase transition in olive of oil up to 0.7 GPa. Journal of American Oil Chemists Society 90: 813813.

15. Kiełczynski M, Szalewski A, Balcerzak K, Wieja AJ, Rostocki RM (2014) Application of ultrasonic wave celerity measurement for evaluation of physicochemical properties of olive oil at high pressure and various temperatures. LWT-Food Science and Technology 57: 253-259.

16. Pawlicki LT, Siegoczyński RM, Ptasznik S, Marszałek K (2021) Electric properties of olive oil under pressure, European Food Research and Technology 247(8): 19331937.

17. Hiroshi M, Maki K, Sachiko T, Masayuki S (2016) Basic Characteristics of Quartz Crystal Sensor with Interdigitated Electrodes. Analytical Chemistry Research 7: $23-30$. 